\title{
A LOCAL CHARACTERIZATION OF DARBOUX $B$ FUNCTIONS
}

\author{
RICHARD G. GIBSON
}

\begin{abstract}
A. M. Bruckner and J. B. Bruckner gave the definition of Darboux $B$ functions and proved a theorem which is a local characterization of real-valued Darboux $B$ functions. The purpose of this paper is to generalize this theorem. To this end, the definition of a function being Darboux $B$ at a point is given which has a metric continuum as its range. Hence, the theorem that a function is Darboux $B$ if and only if it is Darboux $\mathbb{B}$ at each point.
\end{abstract}

1. Introduction. A function having domain and range a subset of the real line is said to be a Darboux function if the image of every connected subset of its domain is a connected set. A detail account of Darboux functions can be found in the survey article by Bruckner and Ceder [2].

A. M. Bruckner and J. B. Bruckner [1] gave the definition of Darboux $\mathbb{B}$ functions which are defined on a euclidean space and having a separable metric space as the range, and proved a theorem which is a local characterization of real-valued Darboux $\mathfrak{B}$ functions. Then they made the statement that it would be of interest to know whether such a theorem exists in case the range is not a subset of the real line. The purpose of this paper is to give an answer to this query. The local condition given by Bruckner and Bruckner [1] was in terms of limit superior and limit inferior. That local condition will be replaced by one where the range of the function is a metric continuum. How much less one can assume about the range without losing the results is an open question.

$\$ 2$ contains some of the needed definitions and a definition of Darboux $B$ at a point which replaces the local condition given in [1]. $\$ 3$ contains the main theorem which is preceded by a lemma.

2. Preliminaries. In this paper $X$ will be a euclidean space, $Y$ a metric continuum, and $B$ a topological base for $X$ such that each member of $B$ is connected. A function $f$ defined on $X$ and having range $Y$ is said to be

Received by the editors February 16, 1974.

AMS (MOS) subject classifications (1970). Primary 54C10; Secondary 26A15.

Key words and phrases. Darboux functions, Darboux $\mathfrak{B}$ functions, Darboux $\mathfrak{B}$ at a point, property $\mathrm{L}$. 
Darboux $B$ provided that if $U$ is in $B$, then $f(\mathrm{cl}(U))$ is connected in $Y$. A base $B$ for $X$ is said to satisfy condition $(*)$ provided any translation of an element of $\mathcal{B}$ is in $\mathcal{B}$. The base $\mathcal{B}$ satisfies condition $(* *)$ provided that for each $x \in X$ and $U \in \mathcal{B}, x \in \mathrm{cl}(U)$, there exists $V \in \mathcal{B}$ such that $x \in \mathrm{cl}(V)$ and $\operatorname{cl}(V)-(x) \subset U$.

Let $f$ be a function defined on $X$ and having range $Y$. The function $f$ is said to have property $\mathrm{L}$ at the point $x$ in $X$ provided that, if $U \in \mathfrak{B}$ and $x$ is a boundary point of $U$, then $f(x)$ is a limit point of $f(U)$. If $x$ is a point in $X$, then a point $y$ in $Y$ is said to be a limit point of $f$ at $x$ provided that there exists a sequence $\left(x_{n}\right)_{n=1}^{\infty}$ of points in $X$ which converges to $x$ such that the sequence $\left(f\left(x_{n}\right)\right)_{n=1}^{\infty}$ converges to $y$.

Definition. Let $X$ be a euclidean space and let $\mathscr{B}$ be a base of connected sets for $X$ which satisfies conditions $(*)$ and $(* *)$. Let $f$ be a function defined on $X$ and having range $Y$. The function $f$ is said to be Darboux $\mathcal{B}$ at the point $p$ in $X$ provided that the following statements are true.

(1) $f$ has property $\mathrm{L}$ at the point $p$ and $f(p)$ is a limit point of $f$ at $p$.

(2) If $U \in \mathcal{B}, p \in U$, and $a$ and $b$ are limit points of $f$ at $p$ relative to $U$, then for each closed subset $C$ of $Y$ which separates $a$ from $b$ there exists $x \in U$ such that $f(x)$ is in $C$.

3. A local characterization. A lemma will be proved before proving the main theorem.

Lemma. Let $f$ be a function defined on $X$ and having range $Y$ and let $\mathcal{B}$ be a basis for $X$ which satisfies conditions $(*)$ and $(* *)$. Let $U \in \mathcal{B}$ and let $f(A)$ and $f(B)$ be two mutually separated sets such that the union of $A$ and $B$ is $\mathrm{cl}(U)$. If $f$ has property $\mathrm{L}$ at each point in $X$ and $P$ is the bound. ary of $A$ relative to $\mathrm{cl}(U)$, then the following statements are true.

(1) $P$ is the boundary of $B$ relative to $\mathrm{cl}(U)$.

(2) $P$ is closed in $X$.

(3) $P \cap U$ is nonempty, dense-in-itself, and of type $G_{\delta}$.

(4) $A \cap P^{\prime}$ and $B \cap P^{\prime}$ are both dense in $P^{\prime}=P \cap U$.

Proof. It is obvious that $P$ is the boundary of $B$ relative to $\mathrm{cl}(U)$ and $P$ is closed in $X$.

To prove that $P \cap U$ is nonempty, note that $P$ is nonempty; for, if $P$ were empty, then $A$ and $B$ would form a separation of the connected set $\operatorname{cl}(U)$. Suppose $P \cap U$ is empty. Then $P \subset \mathrm{cl}(U)-U$, and $U \subset A$ or $U \subset B$. Assume $U \subset A$. Choose any $x$ in $B$. Then $x$ is in $\mathrm{cl}(U)-U$ and $x$ is a boundary point of $U$. Thus $f(x)$ is a limit point of $f(U)$. So $f(x)$ is a limit point of 
$f(A)$ which is in $f(B)$. This contradicts the fact that $f(A)$ and $f(B)$ are mutually separated. Therefore $P \cap U$ is nonempty.

Now suppose $P \cap U$ is not dense-in-itself. Then there is a point $x$ in $P \cap U$, say $x$ is in $P \cap A$, and a $V$ in $B, V \subset U$, such that $P \cap V=(x)$. If $X$ is of dimension greater than or equal to 2 , then $V-(x)$ is connected. So $V-(x) \subset A$ or $V-$ $(x) \subset B$. Since $x$ is in $P, V-(x)$ is not a subset of $A$. So $V-(x) \subset B$. By condition $(* *)$, there is a $W \in \mathcal{B}$ such that $x$ is in $\mathrm{cl}(W)$ and $\mathrm{cl}(W)-(x) \subset V-(x) \subset B$. Since $x$ is a boundary point of $W, f(x)$ is a limit point of $f(W)$. So $f(x)$ is a limit point of $f(B)$ which is in $f(A)$. This is a contradiction. Thus $P \cap U$ is dense-initself if $X$ is of dimension greater than or equal to 2. If the dimension of $X$ is 1 , the result is easily proved.

Since $P$ is closed and $U$ is open, $P \cap U$ is of type $G_{\delta}$.

To prove that $A \cap P^{\prime}$ is dense in $P^{\prime}$, suppose that $A \cap P^{\prime}$ is not dense in $P^{\prime}$. Let $V \subset U$ be an open sphere centered at a point $p \in P^{\prime} \cap B$ such that $V \cap A \cap P^{\prime}$ is empty. Then $A \cap V$ is open and nonempty. Let $x$ be a point of $A \cap V$ such that $d(x, X-V)$ is greater than $2 d(x, p)$ where $d$ is the euclidean metric of $X$. Choose $W \in \mathfrak{B}$ such that $x \in W \subset A \cap V$ and $d(\mathrm{cl}(W), X-V)$ is greater than $d(\mathrm{cl}(W), p)$. Let $q$ be a point of $B$ nearest $x$. Then $q$ is in $V$. Let $W^{\prime}$ be a translation of $W$ such that $q \in \mathrm{cl}\left(W^{\prime}\right)-W^{\prime}$ and $W^{\prime} \subset A$. By condition $(*), W^{\prime} \in \mathcal{B}$. Now choose $W^{\prime \prime} \in \mathcal{B}$ such that $q \in \mathrm{cl}\left(W^{\prime \prime}\right)$ and $\mathrm{cl}\left(W^{\prime \prime}\right)-(q) \subset W^{\prime}$. Now $q \in B$ and $\mathrm{cl}\left(W^{\prime \prime}\right)-(q) \subset A$. Since $q$ is a boundary point of $W^{\prime \prime}$, we have a contradiction by the hypothesis of the theorem. Thus $A \cap P^{\prime}$ is dense in $P^{\prime}$. In a similar way, it can be shown that $B \cap P^{\prime}$ is dense in $P^{\prime}$.

Theorem. Let $X$ be a euclidean space and let $\mathbb{B}$ be a base of connected sets for $X$ which satisfies conditions $(*)$ and $(* *)$. Let $f$ be a function defined on $X$ and having a metric continuum $Y$ as its range. Then $f$ is Darboux $\mathfrak{B}$ if and only if $f$ is Darboux $\mathfrak{B}$ at each point in $X$.

Proof. Suppose $f$ is not Darboux $\mathscr{B}$. Then there exists $U \in \mathfrak{B}$ such that $f(\mathrm{cl}(U))$ is not connected. Let $f(A)$ and $f(B)$ be two mutually separated sets such that the union of $A$ and $B$ is $\operatorname{cl}(U)$.

Let $Y(A)$ and $Y(B)$ be two disjoint open subsets of $Y$ such that $f(A) \subset$ $Y(A)$ and $f(B) \subset Y(B)$. Let $C$ be the complement of $Y(A) \cup Y(B)$. Then $C$ separates $Y(A)$ and $Y(B)$. Let $P$ be the boundary of $A$ in $\operatorname{cl}(U)$ and let $P^{\prime}$ $=P \cap U$. By the lemma both the sets $A \cap P^{\prime}$ and $B \cap P^{\prime}$ are dense in $P^{\prime}$.

We now show that if $p$ is in $A \cap U$ and $W$ is an open set containing $C$, then there exists an open set $V \in \mathscr{B}$ containing $p$ such that if $x \in \mathrm{cl}(V)$, 
then $f(x) \in Y(A) \cup W$. Suppose the condition fails for some $p \in A \cap U$ and $W$ $\supset C$. Let $V_{1}, V_{2}, \cdots$ be a sequence of sets each containing $p$ such that $V_{k} \in \mathcal{B}$ for each $k=1,2, \cdots$ and $\bigcap_{k=1}^{\infty} V_{k}=(p)$. Then for each $k$ there exists $x_{k} \in V_{k}$ such that $f\left(x_{k}\right)$ is not in $Y(A) \cup W$. Since $Y(A) \cup W$ is open and $Y$ is compact, $Y-Y(A) \cup W$. is compact. Thus there exists a subsequence of $\left(f\left(x_{k}\right)\right)_{k=1}^{\infty}$ which converges to some point $y \in Y-Y(A) \cup W$, and hence $y$ is a limit point of $f$ at $p$ which is in $Y(B)$. Since $f(p) \in Y(A)$ and $f(p)$ is a limit point of $f$ at $p$, there exists $x \in U$ such that $f(x) \in C$. This is a contradiction. Similarly, if $p \in B \cap U$ and $W$ is an open set containing $C$, then there exists $V \in \mathbb{B}$ containing $p$ such that if $x \in \mathrm{cl}(V)$, then $f(x) \in$ $Y(B) \cup W$.

Let $W_{1}, W_{2}, \ldots$ be a sequence of open subsets of $Y$ such that $\bigcap_{k=1}^{\infty} W_{k}$ $=C$. Let $p_{1} \in A \cap P^{\prime}$. Choose $V_{1} \in \mathcal{B}$ such that $p_{1} \in V_{1}$, the diameter of $V_{1}<1, \operatorname{cl}\left(V_{1}\right) \subset U$, and if $x \in \mathrm{cl}\left(V_{1}\right)$, then $f(x) \in Y(A) \cup W_{1}$. Since $B \cap P^{\prime}$ is dense in $P^{\prime}$, there is a $p_{2}$ in $B \cap P^{\prime} \cap V_{1}$. Let $V_{2} \in \mathfrak{B}$ such that $p_{2} \in V_{2}$, $V_{2} \subset V_{1}$, the diameter of $V_{2}<1 / 2$, and if $x \in \operatorname{cl}\left(V_{2}\right)$, then $f(x) \in Y(B) \cup W_{2}$. Continuing in this manner, we obtain a sequence of points $\left(p_{k}\right)_{k=1}^{\infty}$ and a sequence of open sets $\left(V_{k}\right)_{k=1}^{\infty}$ such that for each $k$, the following conditions are satisfied.

1. $p_{k} \in V_{k} \cap P^{\prime}, V_{k} \in \mathfrak{B}, V_{k+1} \subset V_{k}$, the diameter of $V_{k}<1 / k$.

2. If $x \in \mathrm{cl}\left(V_{k}\right)$ and $k$ is odd, then $f(x) \in Y(A) \cup W_{k}$.

3. If $x \in \mathrm{cl}\left(V_{k}\right)$ and $k$ is even, then $f(x) \in Y(B) \cup W_{k}$.

Note that the set $\bigcap_{k=1}^{\infty} \mathrm{cl}\left(V_{k}\right)$ consists of a single point, say $p$.

Since $\operatorname{cl}\left(V_{1}\right) \subset U, p \in U$. Since $p \in \operatorname{cl}\left(V_{k}\right)$ for each $k=1,2, \cdots$, it follows that $f(p)$ is in each $W_{k}$. Thus $f(p) \in \bigcap_{k=1}^{\infty} W_{k}$, and so $f(p) \in C$. This is a contradiction, and hence $f$ is Darboux $\mathcal{B}$.

We now assume $f$ is Darboux $\mathcal{B}$ and prove that $f$ is Darboux $\mathcal{B}$ at each point of $X$. The fact that $f$ has property $\mathrm{L}$ of the definition of Darboux $B$ at each point is obvious. We now prove that $f(p)$ is a limit point of $f$ at $p$. Let $\left(W_{k}\right)_{k=1}^{\infty}$ be a descending sequence of open subsets of $Y$ each containing $f(p)$ such that $\bigcap_{k=1}^{\infty} W_{k}=(f(p))$. Let $\left(V_{k}\right)_{k=1}^{\infty}$ be a descending sequence of sets in $B$ each containing $p$ such that $\bigcap_{k=1}^{\infty} V_{k}=(p)$. Since $f$ is Darboux $\mathcal{B}$ and $p \in V_{k}, f\left(V_{k}\right)$ is a connected subset of $Y$ which contains $f(p)$ for each $k=1,2, \cdots$. Now there exists $x_{k} \in V_{k}$ such that $x_{k} \neq p$ and $f\left(x_{k}\right)$ $\epsilon W_{k}$ for each $k=1,2, \ldots$. The sequence $\left(x_{k}\right)_{k=1}^{\infty}$ converges to $p$ and the sequence $\left(f\left(x_{k}\right)\right)_{k=1}^{\infty}$ converges to $f(p)$. Therefore $f(p)$ is a limit point of $f$ at $p$.

We now prove part (2) of the definition of Darboux $\mathcal{B}$ at a point. Let $U$ 
$\in \Re, p \in U$, and let $a$ and $b$ be limit points of $f$ at $p$ relative to $U$. Let $C$ be a closed subset of $Y$ which separates $a$ from $b$. Thus there exists two mutually separated open sets $V$ and $W$ such that $Y-C=V \cup W, a \in V$ and $b \in W$. Since $a$ and $b$ are limit points of $f$ at $p$, there exist $y, z \in U$ such that $f(y) \in V$ and $f(z) \in W$. Thus $C$ separates $f(y)$ and $f(z)$. Since $f(U)$ is connected, there exists $x \in U$ such that $f(x) \in C$, and we are finished.

\section{REFERENCES}

1. A. M. Bruckner and J. B. Bruckner, Darboux transformations, Trans. Amer. Math. Soc. 128 (1967), 103-111. MR 36 \#1586.

2. A. M. Bruckner and J. G. Ceder, Darboux continuity, Jber. Deutsch. Math.Verein. 67 (1964/65), Abt. 1, 93-117. MR 32 \#4217.

3. James Dugundji, Topology, Allyn and Bacon, Boston, Mass., 1967. MR 33 \#1824.

DEPARTMENT OF MATHEMATICS, ALABAMA STATE UNIVERSITY, MONTGOMERY, ALABAMA 36101 